\title{
Revisiting Low-Fidelity Two-Fluid Models for Gas-Solids Transport
}

\author{
Najeem Adeleke $^{\mathrm{a}, *}$, Michael Adewumi ${ }^{\mathrm{a}, *}$, Thaddeus Ityokumbul ${ }^{\mathrm{a}}$ \\ ${ }^{a}$ Department of Energy and Mineral Engineering, The Pennsylvania State University, \\ University Park, PA 16801
}

\begin{abstract}
Two-phase gas-solids transport models are widely utilized for process design and automation in a broad range of industrial applications. Some of these applications include proppant transport in gaseous fracking fluids, air/gas drilling hydraulics, coal-gasification reactors and food processing units. Systems automation and real time process optimization stands to benefit a great deal from availability of efficient and accurate theoretical models for operations data processing. However, modeling two-phase pneumatic transport systems accurately requires a comprehensive understanding of gas-solids flow behavior. In this study we discuss the prevailing flow conditions and present a low-fidelity twofluid model equation for particulate transport. The model equations are formulated in a manner that ensures the physical flux term remains conservative despite the inclusion of solids normal stress through the empirical formula for modulus of elasticity. A new set of Roe-Pike averages are presented for the resulting strictly hyperbolic flux term in the system of equations, which was used to develop a Roe-type approximate Riemann solver. The resulting scheme is stable regardless of the choice of flux-limiter. The model is evaluated by the prediction of experimental results from both pneumatic riser and air-drilling hydraulics systems. We demonstrate the effect and impact of numerical formulation and choice of numerical scheme on model predictions. We illustrate the
\end{abstract}

\footnotetext{
${ }^{*}$ Corresponding authors

Email addresses: najm@psu.edu / nadeleke@gmail.com (Najeem Adeleke), m2a@psu.edu (Michael Adewumi)
}

Preprint submitted to Journal of Computational Physics

May 5, 2016

(C) 2016. This manuscript version is made available under the Elsevier user license http://www.elsevier.com/open-access/userlicense/1.0/ 
capability of a low-fidelity one-dimensional two-fluid model in predicting relevant flow parameters in two-phase particulate systems accurately even under flow regimes involving counter-current flow.

Keywords: approximate Riemann solver, two-fluid model, multiphase, compressible flow, pneumatic conveying, hyperbolic systems

2015 MSC: 00-01, 99-00

\section{Introduction}

Particulate transport in fluid media is common in many industrial processes. Designing such processes to predefined capacity limits can be abstruce and highly dependent on the ability to model and simulate them. Furthermore, 5 real-time mechanistic model simulation coupled to model parameter tuning algorithms that take advantage of available operations data is crucial for efficient process control automation. Process automation frameworks aid the seamless real-time optimization of engineering operations and industrial processes using measured operations data. Stand alone commercial and open-source simulators like FLUENT, Star-CCM+ and OpenFOAM are capable of modeling particulate transport at high fidelity. However, they are inefficient for real-time process optimization and capacity design as they are built with the flexibility for dynamic data utilization while run perpetually on a remote server with little or no human interaction. Hence the need for lower fidelity models that are fast, accurate and robust.

Some well known computational models, such as K-FIX [1], MULTIFIX [2, 3, FORSIM [4, OLGA [5], TACITE [6], PLAC and TUFFP [7, TRAC [8], RELAP [9, 10, 11] and CATHARE [12] are examples of lower fidelity models for multiphase flow systems. However, these tend to suffer from frontal smear20 ing, which ultimately affects parameter predictions when applied to two-phase particulate transport systems. This fact led some authors [13] to the conclusion that such lower fidelity models are incapable of making accurate parameter predictions when applied to two-phase particulate transport systems. 
Toumi and Kumbaro [14] noted that the smearing of the fronts observed in ingly, their two-dimensional simulation results also indicated poor steady-state solids concentration predictions as well. However, they attributed the discrepancy in the numerical predictions relative to experimental measurements to the inaccuracies of the x-ray densitometer used in Luo's experiment [17] to measure 45 the solids concentrations.

Syamlal 20] also presented a new set of two-fluid model equations that are hyperbolic under a wide range of flow conditions by including a bouyant force term that accounts for relative velocity between the fluid and solids phases. He expressed the flow equations using primitive variables and also showed through characteristic analysis, where various sets of flow equations lose hyperbolicity. However, Hou and Le Floch 21] have shown that numerical formulations such as those that employ primitive variables do not converge to the correct solution when discontinuities are present.

Hudson and Harris [22] also presented a high resolution scheme for gas-solids 
two-phase isentropic flow. Both of Gidaspow's [23] non-hyperbolic (Model A) and strictly hyperbolic (Model B) equations were evaluated. Model C, which is also non-hyperbolic was analyzed but not evaluated as it displayed a larger region of non-hyperbolicity relative to Model A. They utilized a conservative finite volume scheme and a Roe-type approximate Riemann solver. They presented a set of Roe-Pike averages for their system of equations and solved these equations using conserved variables. They however reported some instability in their numerical scheme which they attributed to sensitivity to the choice of fluxlimiter used. It should be noted that the observation of numerical instabilities when solving strictly hyperbolic systems, as is the case for model B, reported by Hudson and Harris point to flaws in the numerical scheme itself.

Kamath and Du [24] presented an approximate Riemann solver for granular gas flow proposed by Goldshtein and Shapiro [25]. Kamath and Du presented a Roe-type algorithm for the hyperbolic system, which included nonconservative terms. The non-conservative terms introduce non-isentropic effects in acoustic-wave propagation within granular media and contribute to the Rankine-Hugoniot relations across discontinuities. They reported stable and accurate results for a one-dimensional test case for shocks with the flow features of a fluidized region downstream of the shock and a compacted solid-block region adjacent to the wall. They concluded that the Roe-type scheme they had 75 presented may be relevant in the investigation of two-phase flows.

Ibraheem and Adewumi [26] also studied two-phase gas-solids transport for the initiation stage of hydrate formation and transport in natural gas pipelines. They utilized a conservative finite volume scheme and applied a Roe-type approximate Riemann solver using the set of Roe-Pike averages they had presented. associated with the solids phase completely.

While there has been many studies related to the construction of high resolution schemes for gas-solids two phase flow [20, 22, 24, 27], there has not been enough effort in investigating the overarching benefit of improved resolution ${ }_{85}$ of discontinuous fronts on parameter predictions in gas-solids systems. In this 
study, we present a new set of Roe-Pike averages for a system of strictly hyperbolic two-fluid gas-solids flow model equation of the form of Gidaspow's Type-B model 23. The resulting numerical scheme is insensitive to the choice of fluxlimiters. We also show that the inaccuracies observed in parameter predictions 90 in one-dimensional two-fluid gas-solids models are highly dependent on the numerical formulation and the capability of the numerical method of choice to capture and fully resolve discontinuities in the solution of the model equations.

\section{Background}

Most process optimization scenarios involve minimizing power requirements while maintaining adequate gas flow for particulate transport. This typically translates to minimizing pressure drop in the system while maintaining a particular flow condition or regime suitable for the specific operation. Gas-solid models developed for process automation frameworks need to be accurate under the broad spectrum of flow regimes expected under dilute and dense phase flow conditions but simple enough to minimize computational costs. For a vertical pneumatic transport system, Fig. 1 shows the typical curves of pressure drop as a function of superficial gas velocity at fixed solid mass feed rates $\left(W_{s_{1}}\right.$, $\left.W_{s_{2}}, W_{s_{3}}\right)$ also known as the flow characteristics curve (FCC) or Zenz state diagram [28.

Between $\mathrm{AB}$, fluid-wall viscous/frictional resistance contribution to the pressure drop in the conduit predominates. In this section, annular pressure drop is directly proportional to gas velocity. Therefore, as gas velocity decreases, pressure drop decreases and vice-versa. Region (AB) is also known as the dilute flow region. The flow characteristic behavior of the fluid mixture while within region $\mathrm{AB}$ is similar to the behavior of single phase gas flow $\left(W_{s_{0}}=0\right)$. Between $\mathrm{BC}$, pressure drop due to head loss and cloud particle drag are dominant. In this region, there exists an inverse relationship between superficial gas velocity and pressure drop in the conduit. This is due to the fact that as gas velocity is reduced below a critical value (point B), the rate of solid accumulation in 


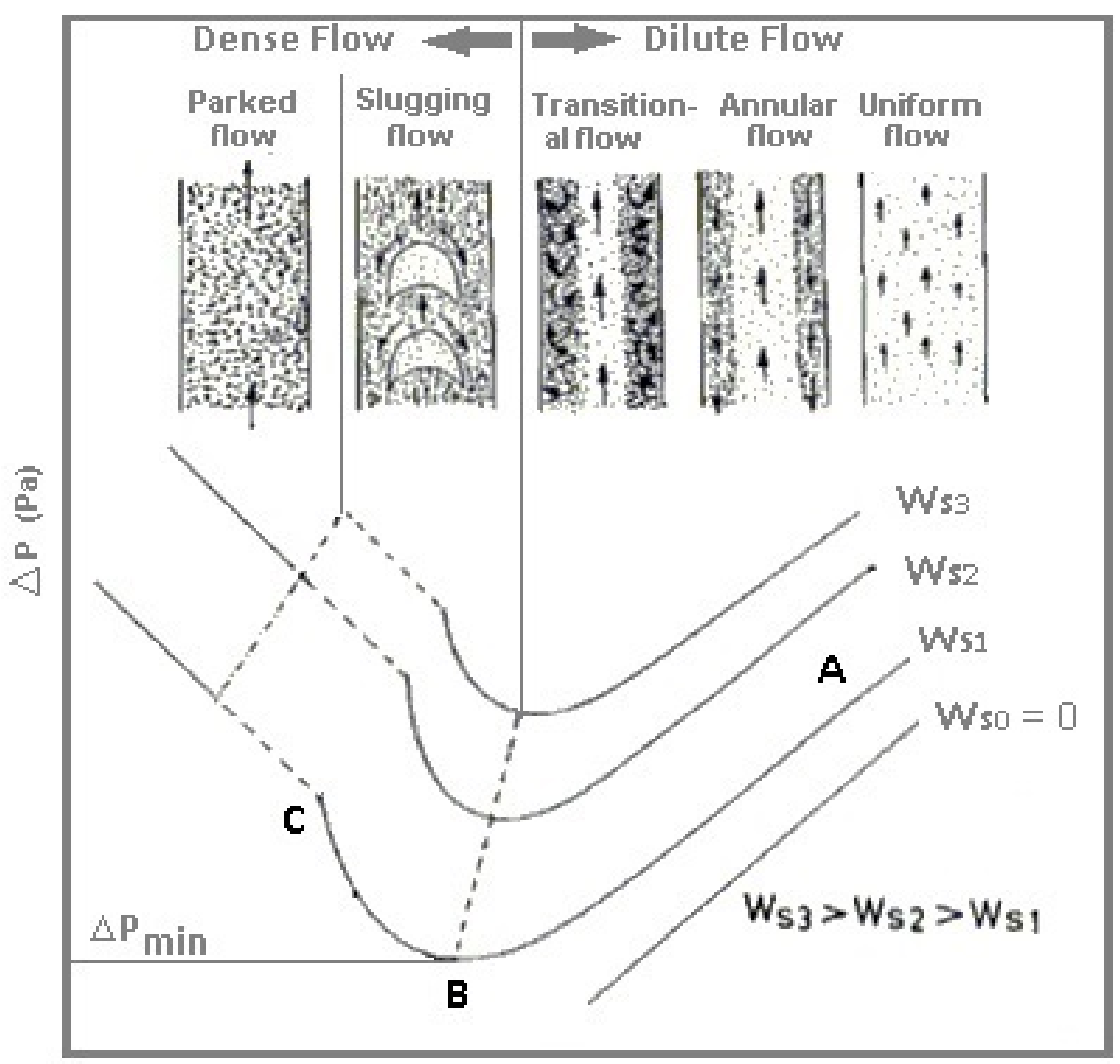

Ug (m/s)

Figure 1: Flow regimes and transitions between dense and dilute phase flow conditions [Modified from Cheremisinoff et al. (1984)]

the conduit is higher, which ultimately translates to higher pressure difference across the conduit as a result of the higher hydrostatic head loss and cloud particle drag effects.

Fig. 1 also shows the various flow regimes experienced under both dilute and dense flow for vertical pneumatic transport. At point A (relatively higher superficial gas velocities), solids concentration is distributed uniformly therefore the flow remains uniform. If superficial gas velocity is reduced (Between A and B), particles cluster around the pipe wall forming annular flow whereby higher 
solids concentration is formed near the walls of the conduit with particles near the wall moving in the opposite direction relative to the gaseous phase (countercurrent flow). At even lower superficial gas velocities, a transitional state is observed whereby the annular regions grows until it closes in on itself (Point B). At even lower superficial gas velocities (Between B and C), we begin to observe slug flow, whereby bubbles of particles moving with same orientation as the gaseous phase struggle to move through particles moving in the opposite direction. Here significant pressure oscillations is usually observed. If superficial gas velocity is lowered to point $\mathrm{C}$, packed flow may be observed.

The idea behind a low-fidelity model is the ability to capture the prevailing flow conditions (i.e. pressure drop, solid concentrations and average phase velocities within the conduit) under varying flow regimes using empirical constitutive relations in a one dimensional two-fluid model.

\section{The Mathematical Model}

A one dimensional isothermal two-phase Eulerian-Eulerian transport equation may be expressed as follows:

$$
\mathbf{U}_{t}+\mathbf{F}(\mathbf{U})_{x}=\mathbf{B}(\mathbf{U})
$$

where:

$$
\begin{gathered}
\mathbf{U}=\left[\begin{array}{c}
\varepsilon \rho_{g} \\
(1-\varepsilon) \rho_{s} \\
\varepsilon \rho_{g} v_{g} \\
(1-\varepsilon) \rho_{s} v_{s}
\end{array}\right], \quad \mathbf{F}(\mathbf{U})=\left[\begin{array}{c}
\varepsilon \rho_{g} v_{g} \\
(1-\varepsilon) \rho_{s} v_{s} \\
\varepsilon \rho_{g} v_{g}^{2}+p_{g} \\
(1-\varepsilon) \rho_{s} v_{s}^{2}+p_{s}
\end{array}\right], \\
\mathbf{B}(\mathbf{U})=\left[\begin{array}{c}
0 \\
0 \\
F_{g g}+F_{w g}-F_{d} \\
F_{g s}+F_{w s}+F_{d}
\end{array}\right]
\end{gathered}
$$

Subscripts $g$ and $s$ represents the gas and dispersed solids phases respectively. $\rho, v$ and $p$ are density, velocity, and pressure. $\varepsilon$ is the gas volume fraction, which 
is constrained by $\varepsilon_{\min }<\varepsilon<1$. The source term (B) contains analytical and empirical relations for determining forces (per unit volume) due to gravity $\left(F_{g i}\right)$, wall friction $\left(F_{w i}\right)$ and fluid-particle $\operatorname{drag}\left(F_{d}\right)$, where $i=g, s$. Eq. 1 contains mass and momentum conservation for both the dispersed and carrier phases. Since solid density $\left(\rho_{s}\right)$ is a known constant, we have six unknowns and four equations so far. However, the pressure of the gas and particulate phases may be expressed as a function of gas density and particle concentration (volume fraction) as follows.

Gas pressure is related to gas density and temperature by the following EOS:

$$
p_{g}=\frac{R T}{M_{g}} \rho_{g}
$$

where $R, M_{g}$ and $T$ are the universal gas constant $\left(R=8.314 \times 10^{3} \frac{\mathrm{J}}{\mathrm{kmolK}}\right)$, molecular mass of the gas phase $\left(M_{\text {air }}=28.97 \frac{\mathrm{kg}}{\mathrm{kmol}}\right)$ and fluid temperature. For this isothermal system, pressure of the carrier phase is directly proportional to its density with the proportionality constant $\frac{R T}{M_{g}}$.

The solid pressure is expressed in terms of a modulus of elasticity, $G(\varepsilon)$, which is assumed to be a function only of the local value of the voidage. The solid pressure gradient represents the normal stresses due to particle-particle interactions. Its inclusion helps to prevent the solid phase from reaching unrealistic low values of void fraction in addition to helping to ensure strict hyperbolicity, which though not necessary for stability is a desirable characteristic. It is expressed as follows:

$$
\nabla p_{s}=G(\varepsilon) \nabla(1-\varepsilon)
$$

In order to maintain the conservative form of the physical flux as expressed in Eq. 1 we re-write Eq. 3 as follows:

$$
\nabla p_{s}=G(\varepsilon) \nabla(1-\varepsilon)=\nabla((1-\varepsilon) G(\varepsilon))-(1-\varepsilon) \nabla G(\varepsilon)
$$

where the last term on the right hand side is the non-conservative contribution, which is evaluated algebraically and treated as a forcing function. 
A number of empirical correlations for the modulus of elasticity, $G(\varepsilon)$ have been proposed and several comparative studies [29, 30] have been carried out to test their efficacy. In this paper we adopt one proposed by Gidaspow and Ettehadieh [31:

$$
G(\varepsilon)=10^{-8.76 \varepsilon+5.43}
$$

Eq. 11 can be expressed as:

$$
\mathbf{U}_{t}+\mathbf{F}(\mathbf{U})_{x}=\mathbf{B}(\mathbf{U})
$$

where:

$$
\begin{gathered}
\mathbf{U}=\left[\begin{array}{c}
\varepsilon \rho_{g} \\
(1-\varepsilon) \rho_{s} \\
\varepsilon \rho_{g} v_{g} \\
(1-\varepsilon) \rho_{s} v_{s}
\end{array}\right], \quad \mathbf{F}(\mathbf{U})=\left[\begin{array}{c}
\varepsilon \rho_{g} v_{g} \\
(1-\varepsilon) \rho_{s} v_{s} \\
\varepsilon \rho_{g} v_{g}^{2}+a^{2} \rho_{g} \\
(1-\varepsilon)\left(\rho_{s} v_{s}^{2}+G(\varepsilon)\right)
\end{array}\right], \\
\mathbf{B}(\mathbf{U})=\left[\begin{array}{c} 
\\
0 \\
F_{g g}+F_{w g}-F_{d} \\
(1-\varepsilon) G(\varepsilon)_{x}+F_{g s}+F_{w s}+F_{d}
\end{array}\right]
\end{gathered}
$$

$a$ is the constant speed of sound determined as follows:

$$
a=\sqrt{\frac{d p_{g}}{d \rho}}=\sqrt{\frac{R T}{M_{g}}}
$$

The gravitational force per unit volume is determined as follows:

$$
F_{g g}=\varepsilon \rho_{g} g, \quad F_{g s}=(1-\varepsilon)\left(\rho_{s}-\rho_{g}\right) g
$$

where $g$ is the gravitational acceleration $\left(9.8 \frac{\mathrm{m}}{\mathrm{s}^{2}}\right)$. The viscous force per unit volume is determined as follows:

$$
F_{w i}=\frac{4}{d}\left(\frac{1}{2} \rho v_{i}^{2}\right) f_{i} \quad i=g, s .
$$

The parameters $d$ and $f$ are the pipe diameter and dimensionless friction factor respectively. For the gas phase friction factor, $f_{g}$, is a function of pipe roughness 
$(\epsilon)$ and Reynolds number (Re):

$$
\operatorname{Re}=\frac{\varepsilon \rho_{g} v_{g} d}{\mu_{g}}
$$

where $\mu_{g}$ in Eq. 10 is the gas viscosity and may be determined from well known correlations (e.g. for dry air see Kadoya et al. [32]) at local temperatures and pressures or determined at operating temperature and assumed constant.

The coefficients of friction for the gas phase, $f_{g}$, is estimated from the fanning equation for laminar flow $(\operatorname{Re}<2100)$ and Chen's 33 friction factor equation for turbulent flow conditions $(\operatorname{Re}>2100)$ as expressed in Eq. 11.

$$
f_{g}= \begin{cases}\frac{16}{\operatorname{Re}} & \text { for } \mathrm{Re}<2100 \\ \frac{1}{16}\left(\log \left[\frac{\left(\frac{\epsilon}{d}\right)}{3.7065}-\frac{5.0452}{\operatorname{Re}} \log \left(\frac{\left(\frac{\epsilon}{d}\right)^{1.1098}}{2.8257}+\frac{5.8506}{\operatorname{Re}^{0.898}}\right)\right]\right)^{-2} & \text { for } \operatorname{Re}>2100\end{cases}
$$

The dispersed phase friction factor, $f_{s}$, is determined by the empirical correlation of Yang [34:

$$
f_{s}= \begin{cases}0.00315 \frac{1-\varepsilon}{\varepsilon^{3}}\left((1-\varepsilon) \frac{v_{T}}{v_{g}-v_{s}}\right)^{-0.979} & \text { for vertical flow } \\ 0.0293 \frac{1-\varepsilon}{\varepsilon^{3}}\left((1-\varepsilon) \frac{v_{g}}{\sqrt{g D_{h}}}\right)^{-1.15} & \text { for horizontal flow }\end{cases}
$$

where terminal velocity of a single particle $\left(v_{T}\right)$ is defined as:

$$
v_{T}=\sqrt{\frac{4}{3} \frac{\left(\rho_{s}-\rho_{g}\right) g d_{p}}{C_{d} \rho_{g}}}
$$

The fluid-particle drag force $\left(F_{d}\right)$ is estimated using Eq. 14. The inter-phase friction coefficient $(\beta)$ is estimated using the drag equation in particle-laden fluid [35, 36, 37] expressed in Eq. 15.

$$
\begin{gathered}
F_{d}=\beta\left(v_{g}-v_{s}\right) \\
\beta=\frac{3}{4} \frac{C_{d}(1-\varepsilon) \rho_{g}\left|v_{g}-v_{s}\right|}{d_{p}} g(\varepsilon)
\end{gathered}
$$

The parameters $d_{p}$ and $C_{d}$ are the weighted average particle diameter and dimensionless single sphere drag coefficient respectively. The voidage function, 
$g(\varepsilon)$, accounts for the effects of neighboring particles on the drag of individual particles. For Wen and $\mathrm{Yu}$ [35, $g(\varepsilon)=\varepsilon^{-2.65}$. Di Felice [36] developed an improved correlation for the voidage function using the empirical correlation of Richardson and Zaki [38, which relates the fluidized flux velocity to the void fraction under varying flow regimes. Di Felice's voidage function is of the form:

$$
g(\varepsilon)=\varepsilon^{\eta}
$$

where:

$$
\eta=3.7-0.65 \exp \left[-\frac{1}{2}\left(1.5-\log R e_{r}\right)^{2}\right] .
$$

Single sphere drag coefficient $\left(C_{d}\right)$ is estimated with the correlation of Turton and Levenspiel [39]:

$$
C_{d}=\left\{\begin{array}{lll}
\frac{24}{\operatorname{Re}_{r}}+\frac{4.152}{\operatorname{Re}_{r}^{0} \cdot 343}+\frac{0.413}{1+16300 \mathrm{Re}_{r}^{-1.09}} & \text { if } & \operatorname{Re}_{r}<1 \times 10^{5} \\
0.44 & \text { if } & \operatorname{Re}_{r}>1 \times 10^{5}
\end{array}\right.
$$

where:

$$
\operatorname{Re}_{r}=\frac{\varepsilon \rho_{g}\left|v_{g}-v_{s}\right| d_{p}}{\mu_{g}}
$$

\subsubsection{Characteristic Analysis}

Eq. 6 may be expressed in it's quasi-linear form as follows:

$$
\mathbf{U}_{t}+\mathbf{A}(\mathbf{U}) \mathbf{U}_{x}=\mathbf{B},
$$

where the flux Jacobian matrix, $\mathbf{A}(\mathbf{U})$, expressed in terms of primitive variables is as follows:

$$
\mathbf{A}=\left[\begin{array}{cccc}
0 & 0 & 1 & 0 \\
0 & 0 & 0 & 1 \\
-v_{g}^{2}+\frac{a^{2}}{\varepsilon} & \frac{a^{2} \rho_{g}}{\varepsilon \rho_{s}} & 2 v_{g} & 0 \\
0 & -v_{s}^{2}+\frac{G(\varepsilon)}{\rho_{s}} & 0 & 2 v_{s}
\end{array}\right]
$$


The eigenvalues and eigenvectors of Eq. 19 and therefore Eq. 6 are as follows:

$$
\begin{gathered}
\Lambda=\left[\begin{array}{cccc}
v_{g}-\frac{a}{\sqrt{\varepsilon}} & 0 & 0 & 0 \\
0 & v_{g}+\frac{a}{\sqrt{\varepsilon}} & 0 & 0 \\
0 & 0 & v_{s}-\sqrt{\frac{G(\varepsilon)}{\rho_{s}}} & 0 \\
0 & 0 & 0 & v_{s}+\sqrt{\frac{G(\varepsilon)}{\rho_{s}}}
\end{array}\right] \\
\kappa_{1,2}=\left[\begin{array}{c}
1 \\
0 \\
v_{g} \mp \frac{a}{\sqrt{\varepsilon}} \\
0
\end{array}\right] \quad \kappa_{3,4}=\left[\begin{array}{c}
\frac{\varepsilon\left(\sqrt{G(\varepsilon)} \pm \sqrt{\rho_{s}}\left(v_{g}-v_{s}\right)\right)^{2}-a^{2} \rho_{s}}{a^{2} \rho_{g}} \\
v_{s} \mp \sqrt{\frac{G(\varepsilon)}{\rho_{s}}} \\
a^{2} \rho_{g} \rho_{s}
\end{array}\right]
\end{gathered}
$$

From characteristic analysis, it can be observed that Eq. 6 has real and distinct eigenvalues and is therefore strictly hyperbolic.

\section{Numerical Method}

The presence of shocks or discontinuities not only pose strict restrictions on the mathematical formulation of hyperbolic problems but also on the discretization techniques and numerical schemes necessary to resolve the physical phenomena accurately. Point wise approximations of the differential form of the conservation equations typically breakdown at discontinuities. This is because conservation laws of the form in Eq. 1 are derived from the integral form:

$$
\frac{d}{d t} \int_{x_{i-\frac{1}{2}}}^{x_{i+\frac{1}{2}}} u(x, t) d x=-\left.f(u(x, t))\right|_{x_{i-\frac{1}{2}}} ^{x_{i+\frac{1}{2}}}
$$

with the assumption that $u$ and $f(u)$ are sufficiently smooth. However, this assumption breaks down when discontinuities are present in the solution. Eq. 22 implies that the rate of change of the substance $(u)$ at any given time $(t)$ is only due to the fluxes at the edges $x_{i \pm \frac{1}{2}}$. 
A numerical discretization approach that is based on solving the integral form of conservation laws and thereby offering a better approximation of the physical system is the Finite Volume Method (FVM). The FVM formulation is obtained by integrating Eq. 22 from $t^{n}$ to $t^{n+1}$ to obtain:

$$
\int_{x_{i-\frac{1}{2}}}^{x_{i+\frac{1}{2}}} u\left(x, t^{n+1}\right) d x-\int_{x_{i-\frac{1}{2}}}^{x_{i+\frac{1}{2}}} u\left(x, t^{n}\right) d x=-\left.\int_{t^{n}}^{t^{n+1}} f(u(x, t))\right|_{x_{i-\frac{1}{2}}} ^{x_{i+\frac{1}{2}}} d t .
$$

The time integral on the right hand side of Eq. 23 cannot be evaluated exactly since the physical flux function, $f\left(u\left(x_{i \pm \frac{1}{2}}, t\right)\right.$, varies with time. Thus the flux function is approximated numerically using an average value, $F_{i \pm \frac{1}{2}}$, within time interval $t^{n}$ to $t^{n+1}$ at the cell interfaces $x_{i \pm \frac{1}{2}}$. Eq. 23 may also be expressed as:

$$
U_{i}^{n+1}=U_{i}^{n}+\frac{\Delta t}{\Delta x}\left(F_{i-\frac{1}{2}}-F_{i+\frac{1}{2}}\right)
$$

where:

$$
\begin{aligned}
& U_{i}^{n}=\frac{1}{\Delta x} \int_{x_{i-\frac{1}{2}}}^{x_{i+\frac{1}{2}}} u\left(x, t^{n}\right) d x \\
& F_{i \pm \frac{1}{2}} \approx \frac{1}{\Delta t} \int_{t^{n}}^{t^{n+1}} f\left(u\left(x_{i \pm \frac{1}{2}}, t\right)\right) d t .
\end{aligned}
$$

$U_{i}^{n}$ is the spatial average of the solution in the grid cell $x_{i} \in\left[x_{i-\frac{1}{2}}, x_{i+\frac{1}{2}}\right]$ at time $t^{n}$.

Eq. 24 is a fully discretized finite volume formulation and applicable to both scalar and systems of conservation laws. However, we may also adopt a semidiscrete formulation by integrating Eq. 1 only along its spatial dimension to obtain the ODE:

$$
\frac{d}{d t} \mathbf{U}(t)=R_{i}(U)
$$

where

$$
R_{i}(U)=\frac{1}{\Delta x}\left(F_{i-\frac{1}{2}}-F_{i+\frac{1}{2}}\right) .
$$

Eq. 25 is a semi-discrete formulation and it permits the use of higher-order time integration methods in propagating the solution in time. It also remains a finite volume formulation if $\mathbf{U}(t)$ is taken to be the average values of the conserved variables in each grid cell $x_{i} \in\left[x_{i-\frac{1}{2}}, x_{i+\frac{1}{2}}\right]$ at time $t$. Note however, that the numerical fluxes $\left(F_{i \pm \frac{1}{2}}\right)$ in Eq. 25 are different from those of Eq. 24. Instead of time averages, $F_{i \pm \frac{1}{2}}$ in Eq. 25 are evaluated at time, $t$. 
The numerical flux averages are sensitive to time step sizes especially in the explicit formulation of Eq. 24. Therefore, in order to remain within the domain of dependence in every block at each time step, the following limitation are applied:

$$
\Delta t=C F L \frac{\Delta x}{\left(\lambda_{\Omega}^{n}\right)_{\max }}
$$

where $\left(\lambda_{\Omega}^{n}\right)_{\max }$ is the maximum $\lambda$ across the spatial domain at $t^{n}$ and the Courant-Friedrichs-Lewy $(C F L)$ number [40] is a dimensionless quantity. The CFL number may be regarded as the ratio of $\left(\lambda_{\Omega}^{n}\right)_{\max }$ to the grid speed $(\Delta x / \Delta t)$ and determined by the choice of numerical scheme employed in approximating the numerical fluxes as well as the choice of numerical integrator in the case of the semi-descrite formulation of $\mathrm{Eq} 25$.

\subsection{Monotone Schemes}

Any choice of numerical flux that satisfies the consistency condition will result in a conservative scheme. The consistency property is met if for a constant value of $U$ whereby $U_{i}=U_{i+1}=U$, then:

$$
F_{i+\frac{1}{2}}=F_{i+\frac{1}{2}}\left(U_{i}, U_{i+1}\right)=F(U) .
$$

A detailed account of numerical schemes for hyperbolic systems is beyond the scope of this study but may be found in the works of Trangenstein [41, LeVeque [42, Toro [43, Barth and Deconinck 44] and Hirsch [45] among others. However, we would discuss the few relevant to the current study.

A tempting but naive approximation for the numerical flux function is the arithmetic average of the fluxes between two neighboring cells that produces a centered 2nd-order scheme. However, the resulting scheme is unconditionally unstable regardless of the size of the time step adopted. Another centered scheme that's similar but conditionally stable for $0<C F L<1$ is the LaxFriedrich (LxF) method:

$$
F_{i+\frac{1}{2}}=\frac{1}{2}\left(F_{i}+F_{i+1}\right)+\frac{1}{2} \frac{\Delta x}{\Delta t}\left(U_{i}-U_{i+1}\right)
$$


The last term on the right hand side is known as the numerical viscosity term. Although the viscous term is necessary for stability, it artificially smears (or spreads) discontinuities in the solution thereby affecting how accurately shocks are captured. The front smearing, also known as numerical diffusion, is typical of most first-order monotone schemes including those that take into account the physics of propagating wave contributions such as flux vector splitting (FVS) methods [46, 47, Kinetic-FVS [48, 49, Godunov-type schemes [50, 51, 52, 53, 54] and other approximate Reimann solvers [55, 56, 57, 58. However, it is important to note that the later are significantly less diffusive relative to the LxF scheme.

Perhaps the most widely accepted of the aforementioned schemes is that of Roe [52, 53, 54]. For this reason, it has enjoyed extensive application and several improvements have been made to resolve a number of its short-comings since it was first introduced. One such short-coming is the generation of unphysical rarefaction shocks, which violate the entropy condition (see [59, 60, 61]). Roe's method solves the non-linear local Riemann problem by approximating it with a linearized cell-edge-average constant-coefficient linear system of the form:

$$
\begin{gathered}
\mathbf{U}_{t}+\tilde{\mathbf{A}} \mathbf{U}_{x}=0, \quad 0<x<L, \quad t>0 \\
\text { I.C.: } \mathbf{U}(x, 0)=\mathbf{U}^{0}(x)=\left\{\begin{array}{l}
U_{L} \text { if } x<x_{0} \\
U_{R} \text { if } x>x_{0},
\end{array}\right.
\end{gathered}
$$

where $\tilde{\mathbf{A}}=\tilde{\mathbf{A}}\left(U_{L}, U_{R}\right)$ is evaluated at cell edges and must satisfy both the consistency and conservation properties expressed as follows:

$$
\begin{aligned}
\tilde{\mathbf{A}}(\mathbf{U}, \mathbf{U}) & =\mathbf{A}(\mathbf{U}) \\
\mathbf{F}\left(\mathbf{U}_{R}\right)-\mathbf{F}\left(\mathbf{U}_{L}\right) & =\tilde{\mathbf{A}}\left(\mathbf{U}_{R}-\mathbf{U}_{L}\right)
\end{aligned}
$$

Roe developed a method for determining the cell-edge-average matrix, $\tilde{\mathbf{A}}$, by introducing an intermediate variable $(\mathbf{Q})$, such that $\mathbf{U}$ and $\mathbf{F}$ can be expressed in terms of $\mathbf{Q}$ with the aid of transformation matrices as follows:

$$
\begin{array}{r}
\Delta \mathbf{U}=\tilde{\mathbf{B}}(\mathbf{Q}) \Delta \mathbf{Q}, \\
\Delta \mathbf{F}=\tilde{\mathbf{C}}(\mathbf{Q}) \Delta \mathbf{Q}
\end{array}
$$


From Eqs. 32 and 33 we obtain the following:

$$
\tilde{\mathbf{A}}=\tilde{\mathbf{C}} \tilde{\mathbf{B}}^{-1} \text {. }
$$

Roe and Pike [54] later presented a more efficient approach to determining $\tilde{\kappa}_{i}, \tilde{\lambda}_{i}$ and $\tilde{\alpha}_{i}$. It involves obtaining what is known as the Roe-Pike averages, which are then used to evaluate $\tilde{\mathbf{A}}$ or $\tilde{\kappa}_{i}, \tilde{\lambda}_{i}$ and $\tilde{\alpha}_{i}$ at cell edges. The numerical flux for Roe scheme with entropy correction typically takes the form:

$$
F_{i+\frac{1}{2}}=\frac{1}{2}\left(F_{i}+F_{i+1}\right)+\frac{1}{2} \sum_{j=1}^{m} \tilde{\alpha}_{j} \psi\left(\tilde{\lambda}_{j}\right) \tilde{\kappa}_{j}
$$

In this study we were able to develop a new set of Roe-Pike averages for the non-linear hyperbolic system expressed in Eq. 6 by introducing the intermediate variable $(\mathbf{Q})$ of the form:

$$
\mathbf{Q}=\left[\begin{array}{c}
\sqrt{\varepsilon \rho_{g}} \\
\sqrt{(1-\varepsilon) \rho_{s}} \\
v_{g} \sqrt{\varepsilon \rho_{g}} \\
v_{s} \sqrt{(1-\varepsilon) \rho_{s}}
\end{array}\right]
$$

The resulting Roe-Pike averages are as follows:

$$
\begin{gathered}
\tilde{\varepsilon}=1-\frac{1}{4}\left(\sqrt{1-\varepsilon_{L}}+\sqrt{1-\varepsilon_{R}}\right)^{2} \\
\tilde{\rho}_{g}=\frac{1}{4 \tilde{\varepsilon}}\left(\sqrt{\varepsilon_{L} \rho_{g_{L}}}+\sqrt{\varepsilon_{R} \rho_{g_{R}}}\right)^{2} \\
\tilde{v}_{g}=\frac{v_{g_{L}} \sqrt{\varepsilon_{L} \rho_{g_{L}}}+v_{g_{R}} \sqrt{\varepsilon_{R} \rho_{g_{R}}}}{\sqrt{\varepsilon_{L} \rho_{g_{L}}}+\sqrt{\varepsilon_{R} \rho_{g_{R}}}} \\
\tilde{v}_{s}=\frac{v_{s_{L}} \sqrt{1-\varepsilon_{L}}+v_{s_{R}} \sqrt{1-\varepsilon_{R}}}{\sqrt{1-\varepsilon_{L}}+\sqrt{1-\varepsilon_{R}}}
\end{gathered}
$$

\subsection{Higher-Order Monotonicity Preserving Schemes}

The disadvantage of the monotone schemes presented here is that they experience smearing or numerical diffusion at discontinuities. Higher-order schemes, which include those of Lax-Wendroff (LW) 62], Richtmyer [63], MacCormack 64, weighted-average flux (WAF) 65, 66] and those based on MUSCL-reconstruction [67, 68, 69, 70, 71], resolve discontinuous fronts better but produce spurious oscillations at sharp gradients. These non-physical oscillations also referred 
to as numerical dispersion exist as numerical artifacts in the solution. In an effort to combine the benefits of higher-order schemes with the monotone nature of first-order schemes, higher-order monotonicity preserving schemes were developed. Schemes based on ensuring the Total Variation Diminishing (TVD) property and Essentially Non-Oscillatory (ENO) type [72, help to limit the numerical dispersion experienced with higher-order schemes.

In this study we apply the following Roe-type higher-order TVD scheme:

$$
F_{i+\frac{1}{2}}=F_{i+\frac{1}{2}}^{R o e}+\Psi^{-}\left(F_{i+\frac{1}{2}}^{R o e}-F_{i}\right)
$$

where:

$$
\begin{gathered}
F_{i+\frac{1}{2}}^{R o e}=\frac{1}{2}\left(F_{i}+F_{i+1}\right)+\frac{1}{2} \sum_{j=1}^{m} \tilde{\alpha}_{j} \psi\left(\tilde{\lambda}_{j}\right) \tilde{\kappa}_{j} \\
\Psi^{-}=\frac{1}{2}\left(\phi_{i+\frac{1}{2}}^{-}-\frac{\phi_{i+\frac{3}{2}}^{-}}{r_{i+\frac{3}{2}}^{-}}\right) \\
r_{i+\frac{1}{2}}^{+}=\frac{F_{i+2}-F_{i+\frac{3}{2}}^{R o e}}{F_{i+1}-F_{i+\frac{1}{2}}^{\text {Roe }}} \\
r_{i+\frac{1}{2}}^{-}=\frac{F_{i-1}-F_{i-\frac{1}{2}}^{\text {Roe }}}{F_{i}-F_{i+\frac{1}{2}}^{\text {Roe }}}
\end{gathered}
$$

$\psi\left(\tilde{\lambda}_{j}\right)$ is the entropy fix [59, 60, 61] and $\phi$ are flux limiters.

All the limiters listed in Table 1 were tested and the resulting HO-TVD Roe-type scheme utilized in this study was found to be stable regardless of the choice of flux-limiter applied.

\subsection{Initial and Boundary Specifications}

Typically, the superficial gas velocity, $v_{g s}$, solids mass flux, $W_{s}$, and solids concentration, $1-\varepsilon_{i n}$, are known at the conduit inlet terminal and can be specified. In addition, the outlet pressure, $p_{\text {out }}$, is also usually known and can also be specified. We have assumed that the conduit was free of solids and under no flow condition for all of the simulation conducted in this study. Initial and boundary conditions where therefore specified as follows: 


\begin{tabular}{cccc}
\hline Limiter Name & Limiter & $\lim _{r \rightarrow \infty} \phi$ & Reference \\
\hline \hline van Leer & $\phi_{v l}(r)=\frac{r+|r|}{1+|r|}$ & 2 & van Leer, 1974 \\
MC*** & $\phi_{m c}(r)=\max [0, \min (2 r, 0.5(1+r), 2)]$ & 2 & van Leer, 1977 \\
van Albada & $\phi_{v a}(r)=\frac{r^{2}+r}{r^{2}+1}$ & 1 & van Albada et al., 1982 \\
Minmod & $\phi_{m m}(r)=\max [0, \min (1, r)]$ & 1 & Roe, 1982 \\
SuperBee & $\phi_{s b}(r)=\max [0, \min (2 r, 1), \min (r, 2)]$ & 2 & Roe, 1983 \\
Osher* & $\phi_{o s}(r)=\max [0, \min (r, \beta)]^{*}$ & $\beta$ & Chakravathy \& Osher, 1983 \\
Sweby & $\phi_{s w}(r)=\max [0, \min (\beta r, 1), \min (r, \beta)]^{*}$ & $\beta$ & Sweby, 1984 \\
Ospre & $\phi_{o p}(r)=\frac{1.5\left(r^{2}+r\right)}{\left(r^{2}+r+1\right)}$ & 1.5 & Waterson \& Deconinck, 1995 \\
\hline \multirow{2}{*}{$1 \leq \beta \leq 2$} & ${ }_{\text {Assymmetric Limiter }}^{* * *}$ Monotonized Central $(M C)$
\end{tabular}

Table 1: Example of limiters from literature

I.C.:

$$
\left[\varepsilon, \rho_{g}, v_{g}, v_{s}\right]_{t=0}=\left\{\begin{array}{lll}
\varepsilon_{\text {in }}, \frac{p_{\text {out }} M_{g}}{R T}, \frac{v_{g s}}{\varepsilon_{\text {in }}}, \frac{W_{s}}{\left(1-\varepsilon_{\text {in }}\right) \rho_{s}} & \text { for } \quad x=0 \\
1, \frac{p_{\text {out }} M_{g}}{R T}, 0, \quad 0 & \text { for } \quad x>0
\end{array}\right.
$$

B.C.:

$$
\left[\begin{array}{c}
\varepsilon \\
v_{g} \\
v_{s} \\
\frac{\partial \rho_{g}}{\partial x}
\end{array}\right]_{x=0}=\left[\begin{array}{c}
\varepsilon_{i n} \\
\frac{v_{g s}}{\varepsilon_{i n}} \\
\frac{W_{s}}{\left(1-\varepsilon_{i n}\right) \rho_{s}} \\
0
\end{array}\right] ; \quad\left[\begin{array}{c}
\frac{\partial \varepsilon}{\partial x} \\
\frac{\partial v_{g}}{\partial x} \\
\frac{\partial v_{s}}{\partial x} \\
p
\end{array}\right]_{x=H}=\left[\begin{array}{c}
0 \\
0 \\
0 \\
p_{\text {out }}
\end{array}\right] ;
$$

where $H$ is the height of the conduit.

\section{Results and Discussion}

We begin by replicating the one-dimensional simulation conducted by Tsuo and Gidaspow [13. They simulated the vertical transport of 520 micron diameter glass beads with density of $2.62 \mathrm{~g} / \mathrm{cm}^{3}$ using air flowing at superficial gas velocity of $5 \mathrm{~m} / \mathrm{s}$ and a solids flux of $25 \mathrm{~kg} / \mathrm{m}^{2} / \mathrm{s}$ in a grounded $7.62 \mathrm{~cm}$ diameter acrylic plastic pipe. Boundary specifications were as follows:

$$
\left[\begin{array}{c}
1-\varepsilon_{\text {in }} \\
v_{\text {gs }} \\
W_{s} \\
p_{\text {out }}
\end{array}\right]=\left[\begin{array}{c}
0.0246 \\
4.979 \frac{\mathrm{m}}{\mathrm{s}} \\
25 \frac{\mathrm{kg}}{\mathrm{m}^{2} \mathrm{~s}} \\
1 \mathrm{~atm}
\end{array}\right]
$$



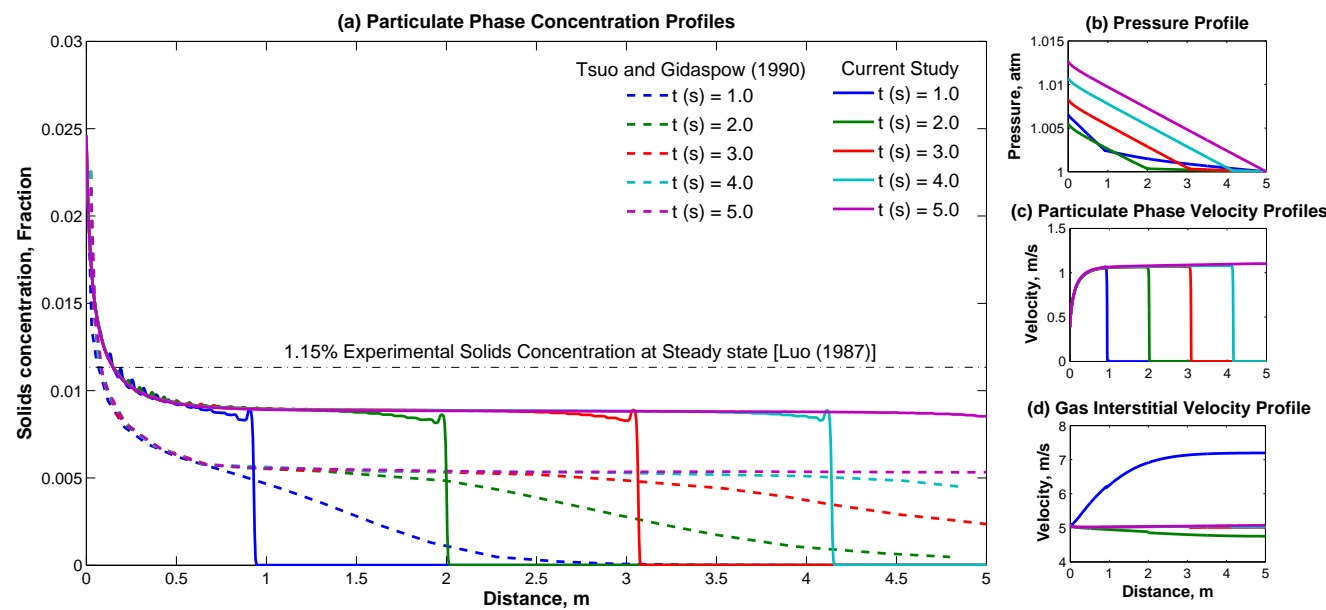

Figure 2: One-dimensional transient simulation of vertical gas-solids flow [Tsuo and Gidaspow (1990)]

Fig. 2 (a) shows the computed solids concentration profile at one second intervals. The dashed lines are simulation results from Tsuo and Gidaspow [13] while solid lines were generated using the HO-TVD Roe scheme from the current study. Figs. 2 (b-d) represent the corresponding pressure and solids velocity and gas interstitial velocity profiles respectively. Fig. 2 (b) illustrate the effect of the invasion of solids on pressure drop before the entire conduit was completely invaded by solids. Fig. 2 (c) illustrates the corresponding velocity of the invading solids as they propagate along the conduit. And Fig. 2 (d) illustrates the turbulent changes in gas velocity at the early time period prior to its stabilization after about 3 seconds.

Tsuo and Gidaspow [13] reported their computed solids concentration in the fully developed region under steady-state flow is 0.0065 . This result shows a 43.5 $\%$ prediction error relative to Luo's [17] measured experimental data. Computed solids concentration in the fully developed region from the current study is 0.0088 indicating a $23.5 \%$ error relative to Luo's measured data. However, considering the comments from Tsuo and Gidaspow [13] about the inaccuracies of the x-ray densitometer utilized by Luo for his measurements, we have decided to estimate the solids concentration at steady-state analytically. 
Under steady-state flow, the mass flux of both gas and particulate phases $\left(W_{g}\right.$ and $\left.W_{s}\right)$ as well as slip velocity $\left(v_{\text {slip }}\right)$ between phases may be assumed constant. Therefore, the slip velocity under steady-state flow may be expressed as follows:

$$
v_{s l i p}=v_{g}-v_{s}=\frac{W_{g}}{\varepsilon \rho_{g}}-\frac{W_{s}}{(1-\varepsilon) \rho_{s}}
$$

The slip velocity may also be roughly estimated using the terminal velocity $\left(v_{T}\right)$ of individual particles [73] for relatively low solids concentrations as follows:

$$
v_{T}=\sqrt{\frac{4}{3} \frac{d_{p}\left(\rho_{s}-\rho_{g}\right) g}{\rho_{g} C_{d}}}
$$

Setting $v_{\text {slip }}$ to $v_{T}$ and combining the expression in Eqs. 42 and 43 , we obtain the following quadratic expression:

$$
\varepsilon^{2}-\left(1+\frac{W_{g}}{\rho_{g} v_{T}}+\frac{W_{s}}{\rho_{s} v_{T}}\right) \varepsilon+\frac{W_{g}}{\rho_{g} v_{T}}=0
$$

In order to ensure $0<\varepsilon<1$, Eq. 44 may be solved to obtain the steady-state solids concentration $\left(1-\varepsilon_{s s}\right)$ as follows:

$$
\varepsilon_{s s}=\frac{1}{2}\left(-b-\sqrt{b^{2}-4 c}\right)
$$

where:

$$
\begin{aligned}
& b=-\left(1+c+\frac{W_{s}}{r h o_{s} v_{T}}\right) \\
& c=\frac{W_{g}}{\rho_{g} v_{T}} \approx \frac{v_{g s}}{v_{T}}
\end{aligned}
$$

Using the analytical expression in Eq. 45, we obtain a steady-state concentration of 0.0091. Comparing the solids concentrations obtained from simulation to that obtained from the analytical expression, we find that Tsuo and Gidaspow [13] were off by $22.6 \%$. However, the solids concentration prediction from the current study were within $2.6 \%$ of the estimated value.

The accuracy of the HO-TVD scheme even for flow conditions experiencing annular counter-current flow lies not only in its capacity to capture discontinuous fronts. The improvement in accuracy observed in this study is also due to the conservative finite volume formulation (Eqs. 24 and 25) employed. This 
is because we are not computing the primitive variables $\left(\varepsilon, \rho_{g}, v_{g}, v_{s}\right)$ directly,

but rather we compute the volume averages of the conserved variables and we later decompose them into the primitive variables seen in Fig. 2, This way we are somewhat implicitly accounting for counter-current flow through the volume average formulation, which ensures that the conserved variables remain conserved. The application of this numerical approach as a building block for two- and three-dimensional models for compressible multiphase flow problems would significantly improve the accuracy of such high-fidelity models as well. However, it is important to note that the conservative formulation alone is not sufficient for model prediction accuracy.
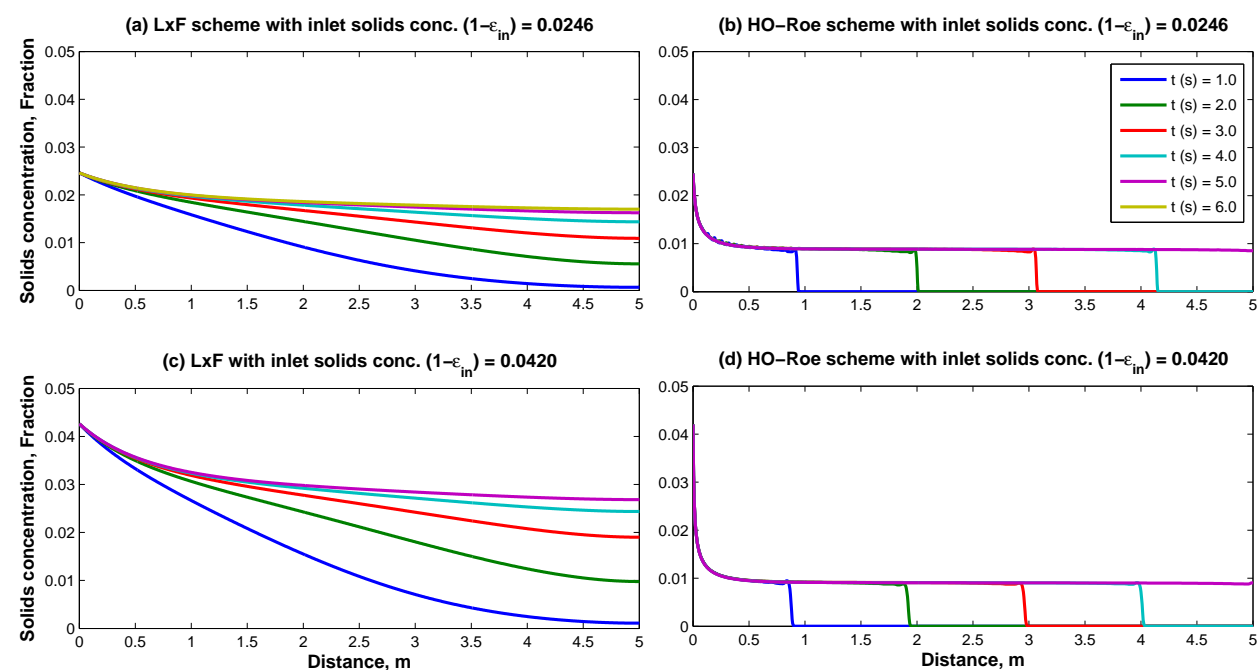

Figure 3: Solids concentration profiles at difference inlet specifications using $\operatorname{LxF}$ (a \& c) and HO-TVD Roe (b \& d) schemes

In order to further illustrate the effect of the numerical scheme on solids 255 concentration predictions we simulate the same conditions specified previously using the highly diffusive Lax-Friedrich (LxF) scheme. Fig. 3 (a) shows the computed solids concentration profiles at one second interval obtained using the LxF scheme. Solid concentration was over-predicted by the LxF scheme with $47.8 \%$ and $68.7 \%$ error relative Luo's measurement and the analytical 
expression of Eq. 45 respectively. Fig. 3 (b) is exactly the same as Fig. 2 (a) and only included in Fig. 3 for comparison.

Furthermore, we test the sensitivity of computed solids concentration in the fully developed region to the specified inlet solids concentration. Fig. 3 (c and d) show simulation results with inlet solids concentration of 0.042 while keeping the total mass flux of solids $\left(W_{s}\right)$ and superficial gas velocity $\left(v_{g s}\right)$ unchanged. It can be observed that the computed solids concentration in the fully developed region obtained using the dissipative LxF scheme is significantly influenced by the inlet specification. However, the opposite is the case for the solutions obtained using the HO-TVD Roe scheme. This is not surprising due to the fact that the total amount of solids entering the conduit remained the same for all results in Fig. 3. The inlet solids concentration specified would only affect the inlet phase velocities to ensure that the total mass flux of solids $\left(W_{s}\right)$ does not change under the specified superficial gas velocity $\left(v_{g s}\right)$ as shown in Section 4.3 under boundary specifications. Therefore, the steady-state solids concentration in the fully developed region should not be influenced by inlet solids concentration if the solids mass flux and superficial gas velocity specified at the inlet remains the same. This concept may also be explained through the analytical expression for steady-state solids concentration (Eq. 45). It can be observed that $\varepsilon_{s s}$ is dependent on $W_{s}$ and $v_{g s}$ but not a function of the inlet solids concentration.

The criticism of Luo's measurement technique was that it was inaccurate under annular flow regime where the solids concentration in the center of the conduit drops significantly below solids concentrations in the near-wall region. However, Luo's measurement technique would be relatively accurate in regions where annular segregation is not likely. An example of that would be the pipe inlet region where the particulate phase is still fully dispersed as they are injected into the acrylic pipe. Therefore, we decided to investigate the accuracy of the current model in predicting void fraction at the pipe inlet region. The system is similar to that investigated in Tsuo and Gidaspow's study however, 
the boundary specifications were as follows:

$$
\left[\begin{array}{c}
\varepsilon_{\text {in }} \\
v_{\text {gs }} \\
W_{s} \\
p_{\text {out }}
\end{array}\right]=\left[\begin{array}{c}
0.975 \\
11.2 \frac{\mathrm{m}}{\mathrm{s}} \\
34.81 \frac{\mathrm{kg}}{\mathrm{m}^{2} \mathrm{~s}} \\
1.068 \mathrm{~atm}
\end{array}\right]
$$
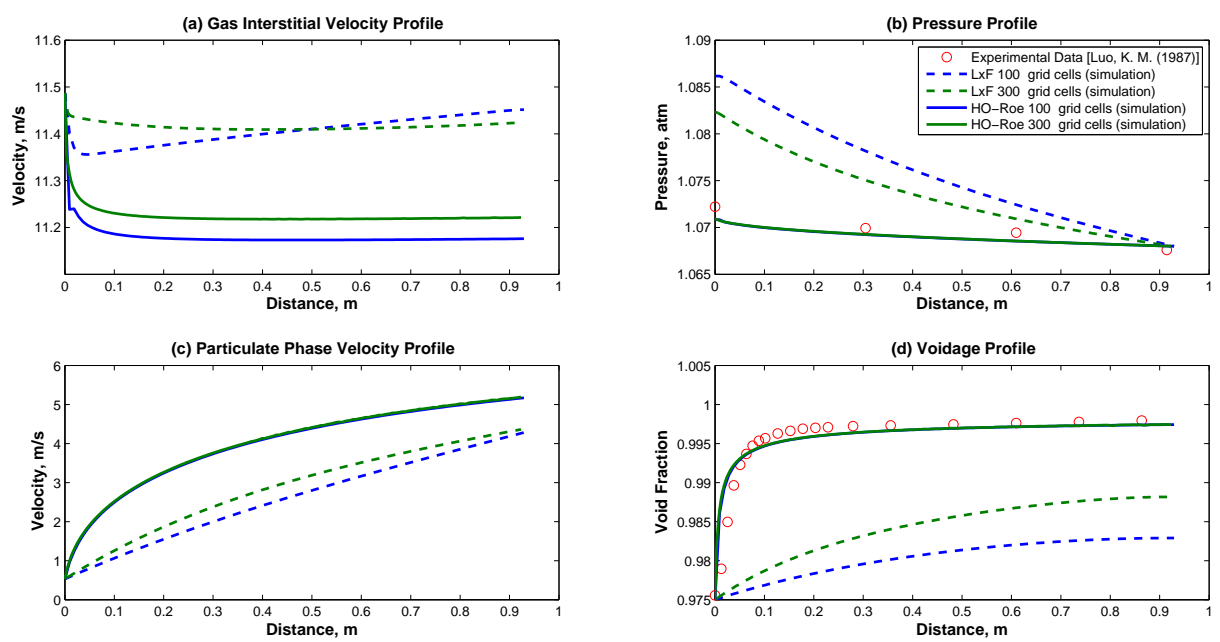

Figure 4: Inlet profiles at steady-state [Luo (1987)]

Fig. 4 (a-d) represent gas velocity, pressure, solids velocity and void fraction $(\varepsilon)$ profiles respectively. Figs. 4 (b and d) illustrate the agreement between the results obtained using the HO-TVD Roe scheme and Luo's measurement.

However, the results obtained using the dissipative LxF scheme showed poor agreement with Luo's measurement even for a refined grid size of 300 grid cells (or blocks) for the less than one meter inlet section. It can also be observed that 100 grid cells were more than sufficient for simulating the inlet section of the pipe when using the HO-TVD Roe scheme since there seems to be barely any noticeable change in the voidage profile when the number of grid cells were increased to 300 .

An additional experimental dataset for dry air drilling operations conducted in the Drilling Hydraulics Research Center at The Pennsylvania State University 
between 1990 and 2003 was also studied. Temple et al. [74, 75] set out to

determine the optimum circulation air velocity for efficient wellbore cleaning. They obtained flow characteristic curves (FCCs) under constant solid mass feed rates and fixed average particle sizes in a closed loop system. They concluded that the optimum annular flow velocity corresponds to the point of minimum pressure differential in their FCC plots.

300

Their experimental results indicated that solids volume fraction (or solids concentration) depends primarily on annular air velocity and solids mass feed rate, which is in concurrence with the findings this study. Their results also show that the steady-state solids concentration in the fully developed region is independent of particle size. However, they showed that the point of minimum pressure drop on the FCC plot is highly dependent on particle diameter, and to a lesser degree, on solids mass feed rate. Figs. 5 and 6 show a comparison of our simulation with Temple's [74, 75] experimental data around the region of minimum pressure difference where counter-current flow may be observed. In order to use the current one-dimensional model to simulate annular flow we defined the conduit hydraulic diameter as the difference between the wellbore ID and drill pipe OD [76]. Solids mass feed rate, $W_{s} \approx 15 \frac{g}{s}$ and volume averaged particle diameter, $\bar{d}_{p} \approx 346.5 \mu \mathrm{m}$ for both simulations. Boundary specifications were defined as follows:

Simulation 1:

$$
\left[\begin{array}{c}
\varepsilon_{\text {in }} \\
v_{\text {gs }} \\
W_{s} \\
p_{\text {out }}
\end{array}\right]=\left[\begin{array}{c}
0.998 \\
7.22 \frac{\mathrm{m}}{\mathrm{s}} \\
15.29 \frac{\mathrm{g}}{\mathrm{s}} \\
0.9568 \mathrm{~atm}
\end{array}\right]
$$

Simulation 2:

$$
\left[\begin{array}{c}
\varepsilon_{\text {in }} \\
v_{\text {gs }} \\
W_{s} \\
p_{\text {out }}
\end{array}\right]=\left[\begin{array}{c}
0.998 \\
6.15 \frac{\mathrm{m}}{\mathrm{s}} \\
15.4 \frac{\mathrm{g}}{\mathrm{s}} \\
0.9541 \mathrm{~atm}
\end{array}\right]
$$



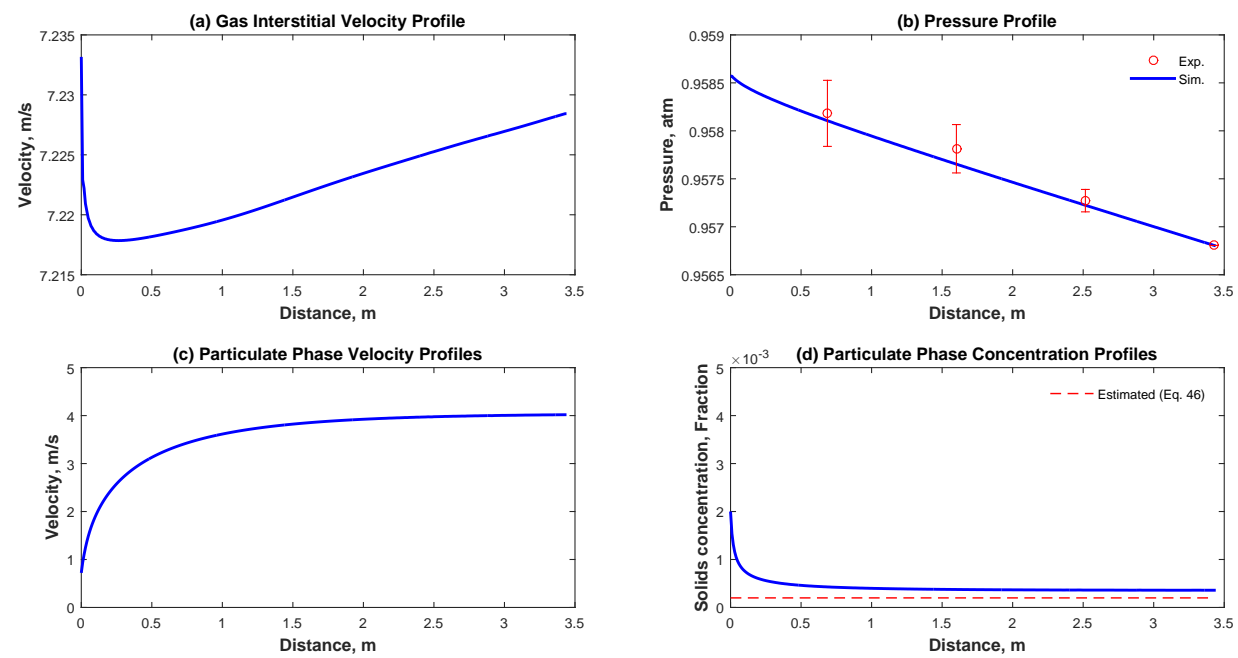

Figure 5: Inlet profiles at steady-state for Simulation 1
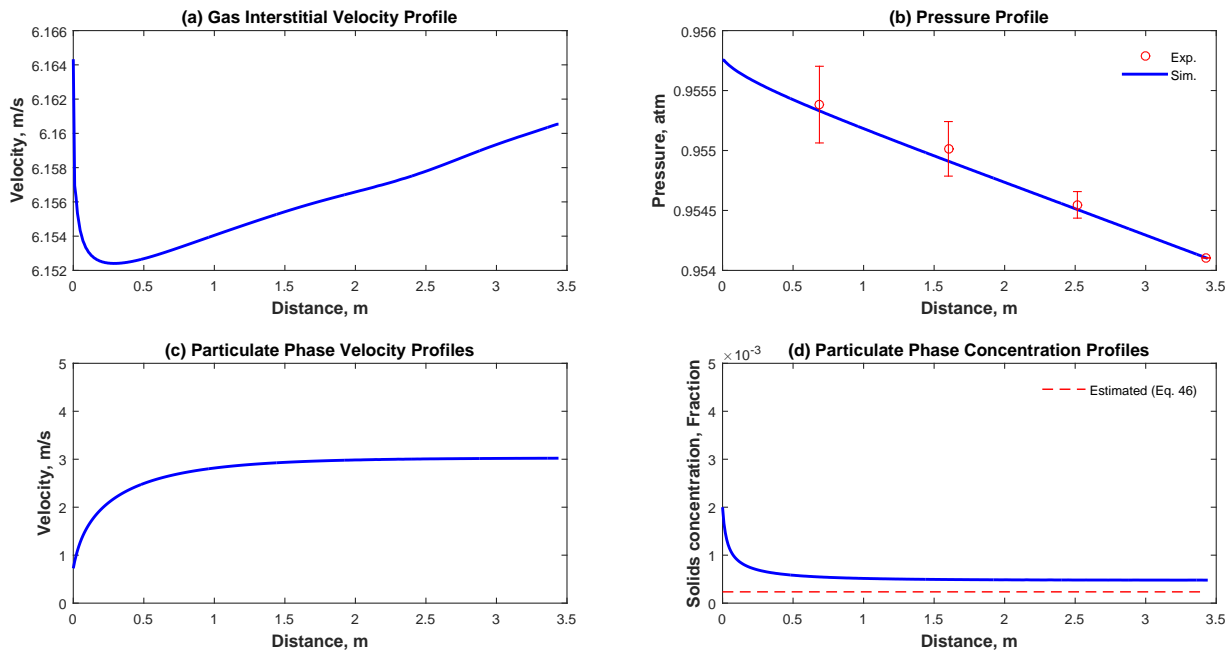

Figure 6: Inlet profiles at steady-state for Simulation 2

Temple [75] reported that they experienced small fluctuations in the particle flow rates due to operational limitations of the L-valve used to introduce solids into the wellbore. The sensitivity of the diaphragm-type differential pressure manometers combined with the flow fluctuations created significant pressure oscillations during their experiments. Although the observed oscillation were 
somewhat dampened by their use of sintered brass inserts in the pressure taps, Temple recorded an experimental error of $\pm 25 \%$ for their differential pressure readings. Temple et al. [74, 75] also reported that their recorded solids concentrations were estimated using the following expression:

$$
(1-\varepsilon)=\frac{q_{s}}{q_{s}+q_{g}}
$$

where $q_{s}$ and $q_{g}$ represent solids volumetric rate and annular air flow rates

315 a Roe-type approximate Riemann solver that is stable regardless of the choice of flux-limiter applied. A higher-order total variation diminishing (HO-TVD) Roe-type scheme was used to compute the solution of the two-fluid system using a conservative finite volume formulation. An analytical expression for steadyinferred from the analysis presented here: 
1. The higher-resolution conservative scheme based on the computation of volume averages of the conserved variables employed in this study helped to improve the accuracy of model predictions significantly even for regimes where counter-current flow is present.

2. Solids concentration at steady state is primarily dependent on solids mass feed rate and superficial gas velocity.

3. Solids concentration in the fully developed region is independent of inlet solids concentration.

4. Grid-refinement requirements were reduced when the HO-TVD Roe-type scheme is used compared to more dissipative schemes like Lax-Friedrich.

5. The numerical approach presented here would serve as a good building block for two- and three-dimensional forms of gas-solids two-phase flow modeling.

\section{Future Studies}

While we have illustrated the accuracy of the use of conservative variables and the HO-TVD scheme in improving model predictions in low-fidelity models such as the one-dimensional isothermal two-fluid model for pneumatic conveying, there is still much to be studied. Most importantly, the current study focuses primarily on vertical pneumatic conveying. However, this model is most likely insufficient for transient flow studies in horizontal and angular gas-solids flow systems due to solids deposition. Solids deposition produces a different kind of segregated flow phenomena that can neither be resolved simply by highresolution schemes nor the computation of volume averages of conserved variables. This is because it involves the static accumulation of solids along sections of the conduit, which is different from the segregated flow observed in vertical pneumatic conveying systems.

Another important area of study not addressed here is supercritical fluid conveying. This is when the carrier fluid is allowed to transition into supercritical phase as would be observed when fluids like $\mathrm{CO}_{2}$ or $\mathrm{N}_{2}$ are used in 
conveying proppants downhole while pressurizing a hydrocarbon reservoir during hydraulic fracking. Here, a thermodynamic model must be used to account for the properties of the carrier phase as transitions from gas to gas-like and/or liquid-like supercritical fluid.

Futhermore, the current study is focused on isothermal conveying conditions. However, this assumption might be inappropriate for certain practical applications of gas-solids flow.

\section{Acknowledgment}

This research was sponsored by Penn State, Department of Energy and Mineral Engineering in the College of Earth and Mineral Sciences.

\section{References}

[1] W. Rivard, M. Torrey, K-fix: A computer program for transient, Twodimensional, Two-Fluid Flow (Report LA-NUREG-6623, Los Alamos National Laboratory, Los Alamos, 1977).

[2] M. Syamlal, Multiphase hydrodynamics of gas-solids flow, Ph.D. thesis, Illinois Institute of Technology, Chicago (1985).

[3] M. Syamlal, W. Rogers, T. J. OBrien, Mfix documentation: Theory guide, National Energy Technology Laboratory, Department of Energy, Technical Note DOE/METC-95/1013 and NTIS/DE95000031.

[4] H. Arastoopour, P. Pakdel, M. Adewumi, Hydrodynamic analysis of dilute gassolids flow in a vertical pipe, Powder Technology 62 (2) (1990) 163 170. doi:http://dx.doi.org/10.1016/0032-5910(90)80080-I.

[5] K. H. Bendiksen, D. Maines, R. Moe, S. Nuland, et al., The dynamic two-fluid model olga: Theory and application, SPE production engineering 6 (02) (1991) 171-180. 
[6] C. Pauchon, H. Dhulesia, et al., Tacite: A transient tool for multiphase pipeline and well simulation, in: SPE Annual Technical Conference and Exhibition, Society of Petroleum Engineers, 1994.

[7] F. Vigneron, C. Sarica, J. Brill, Experimental analysis of imposed twophase flow transients in horizontal pipelines, in: 7th International Conference, Multiphase, Vol. 95, 1995, pp. 199-217.

[8] NREG/CR-3858, L.A-10157-MS, 1986 (unpublished).

[9] V. H. Ranson, NUREG/CR-1826, 1982 (unpublished).

[10] V. Ransom, R. Wagner, J. Trapp, Relap5 two-phase fluid model and numerical scheme for economic lwr system simulation, Tech. rep., Idaho National Engineering Lab., Idaho Falls (USA) (1981).

[11] V. Ransom, R. Wagner, J. Trapp, The relap5 two-phase fluid model and numerical scheme for economic lwr system simulation, in: Transient twophase flow, 1983.

[12] J. C. Micaelli, 87-58, CEA, France, 1987 (unpublished).

[13] Y. P. Tsuo, D. Gidaspow, Computation of flow patterns in circulating fluidized beds, AIChE Journal 36 (6) (1990) 885-896.

[14] I. Toumi, A. Kumbaro, An approximate linearized riemann solver for a two-fluid model, Journal of Computational Physics 124 (2) (1996) 286-300. doi:http://dx.doi.org/10.1006/jcph.1996.0060.

[15] D. Drew, L. Cheng, R. Lahey, The analysis of virtual mass effects in twophase flow, International Journal of Multiphase Flow 5 (4) (1979) 233-242.

[16] R. Lahey, L. Cheng, D. Drew, J. Flaherty, The effect of virtual mass on the numerical stability of accelerating two-phase flows, International Journal of Multiphase Flow 6 (4) (1980) 281-294. 
[17] K. M. Luo, Dilute, dense-phase and maximum solids-gas transport, Ph.D. thesis, Illinois Institute of Technology, Chicago (1987).

[18] K. M. Luo, Experimental gas-solid vertical transport, Tech. rep., Illinois Inst. of Tech., Chicago (USA). Dept. of Chemical Engineering (1987).

[19] D. Gidaspow, Y. Tsuo, K. Luo, Computed and experimental cluster formation and velocity profiles in circulating fluidized beds, 1989.

[20] M. Syamlal, A hyperbolic model for fluid-solids two-phase flow, Chemical Engineering Science 66 (19) (2011) 4421-4425.

425 [21] T. Y. Hou, P. G. Le Floch, Why nonconservative schemes converge to wrong solutions: error analysis, Math. Comput. 62 (206) (1994) 497-530. doi: $10.2307 / 2153520$

URL http://dx.doi.org/10.2307/2153520

[22] J. Hudson, D. Harris, A high resolution scheme for eulerian gassolid twophase isentropic flow, Journal of Computational Physics 216 (2) (2006) 494 - 525. doi:http://dx.doi.org/10.1016/j.jcp.2005.12.010 URL http://www.sciencedirect.com/science/article/pii/ S0021999105005620

[23] D. Gidaspow, Hydrodynamics of fiuidizatlon and heat transfer: Supercomputer modeling, Applied Mechanics Reviews 39 (1) (1986) 1-23.

[24] H. Kamath, X. Du, A roe-average algorithm for a granular-gas model with non-conservative terms, Journal of Computational Physics 228 (21) (2009) 8187 - 8202. doi:http://dx.doi.org/10.1016/j.jcp.2009.08.005. URL http://www.sciencedirect.com/science/article/pii/ S0021999109004422

[25] A. Goldshtein, M. Shapiro, Mechanics of collisional motion of granular materials. part 1. general hydrodynamic equations, Journal of Fluid Mechanics $282(1995) 75-114$. 
[26] S. Ibraheem, M. Adewumi, J. Savidge, Numerical simulation of hydrate transport in natural gas pipeline, Journal of energy resources technology 120 (1) (1998) 20-26.

[27] D. Mao, J. R. Edwards, A. V. Kuznetsov, R. K. Srivastava, Development of low-diffusion flux-splitting methods for dense gas-solid flows, Journal of Computational Physics 185 (1) (2003) 100-119.

[28] F. A. Zenz, Two-phase fluid-solid flow, Industrial \& Engineering Chemistry 41 (12) (1949) 2801-2806.

[29] M. Massoudi, K. Rajagopal, J. Ekmann, M. Mathur, Remarks on the modeling of fluidized systems, AIChE Journal 38 (3) (1992) 471-472. doi:10.1002/aic.690380317 URL http://dx.doi.org/10.1002/aic.690380317

[30] D. Patil, M. van Sint Annaland, J. Kuipers, Critical comparison of

1 hydrodynamic models for gas-solid fluidized beds - Part I : bubbling gassolid fluidized beds operated with a jet, Chemical Engineering Science 60 (1) (2005) 57 - 72. doi:10.1016/j.ces.2004.07.059

so URL http://www.sciencedirect.com/science/article/pii/ S0009250904005007

[31] D. Gidaspow, B. Ettehadieh, Fluidization in two-dimensional beds with a jet. 2. hydrodynamic modeling, Industrial \& Engineering Chemistry Fun-

(1) damentals 22 (2) (1983) 193-201. arXiv:http://pubs.acs.org/doi/pdf/ 10.1021/i100010a008, doi:10.1021/i100010a008

URL http://pubs .acs .org/doi/abs/10.1021/i100010a008

[32] K. Kadoya, N. Matsunaga, A. Nagashima, Viscosity and thermal conductivity of dry air in the gaseous phase, Journal of physical and chemical reference data 14 (4) (1985) 947-970.

[33] N. H. Chen, An explicit equation for friction factor in pipe, Industrial \& Engineering Chemistry Fundamentals 18 (3) (1979) 296-297. 
arXiv:http://pubs.acs.org/doi/pdf/10.1021/i160071a019, doi:10. 1021/i160071a019.

URL http://pubs .acs .org/doi/abs/10.1021/i160071a019

[39] R. Turton, O. Levenspiel, A short note on the drag correlation for spheres, Powder Technology 47 (1) (1986) 83 - 86. doi : 10.1016/0032-5910(86)80012-2. 0032591086800122

[40] R. Courant, K. Friedrichs, H. Lewy, On the partial difference equations of mathematical physics, IBM Journal of Research and Development 11 (2) (1967) 215-234. 
[41] J. A. Trangenstein, Numerical solution of hyperbolic partial differential equations, Cambridge University Press, 2009.

[42] R. J. LeVeque, Finite volume methods for hyperbolic problems, Vol. 31, Cambridge university press, 2002.

[43] E. F. Toro, Riemann solvers and numerical methods for fluid dynamics: a

[44] T. J. Barth, H. Deconinck, High order methods for computational physics, Vol. 9, Springer, 1999.

[45] C. Hirsch, Numerical computation of internal and external flows, volume 2: Computational methods for inviscid and viscous flows, vol. 2 (1990). gasdynamic equations with application to finite-difference methods, Journal of Computational Physics 40 (2) (1981) 263 - 293. doi:10.1016/0021-9991(81)90210-2. URL http://www.sciencedirect.com/science/article/pii/ 0021999181902102

[47] B. van Leer, Flux-vector splitting for the euler equations Technical Report, NASA Langley Research Center.

URL http://ntrs .nasa.gov/search.jsp?R=19840052563

[48] J. Mandal, S. Deshpande, Kinetic flux vector splitting for euler equations, Computers \& Fluids 23 (2) (1994) $447 \quad$ - 478. doi : 10.1016/0045-7930(94)90050-7.

a URL http://wWw.sciencedirect.com/science/article/pii/ 0045793094900507

[49] N. Anil, N. Rajan, S. Deshpande, Modified kinetic flux vector splitting 525 (m-kfvs) method for compressible flows, Computers \& Fluids 48 (1) (2011) 137 - 149. doi:10.1016/j.compfluid.2011.04.005. 
URL http://www.sciencedirect.com/science/article/pii/ S004579301100137X

[50] S. K. Godunov, A finite difference method for the computation of discontinuous solutions of the equations of fluid dynamics, Mathematicheckiy sbornik 47 (1959) 357-393.

[51] S. K. Godunov, A difference scheme for numerical solution of discontinuous solution of hydrodynamic equations, Mat. Sb. 47 (1969) 271-306, (translated, US Joint Publ. Res. Service, JPRS 7226).

535 [52] P. Roe, Approximate riemann solvers, parameter vectors, and difference schemes, Journal of Computational Physics 43 (2) (1981) 357 - 372. doi:10.1016/0021-9991(81)90128-5. URL http://www.sciencedirect.com/science/article/pii/ 0021999181901285

540 [53] P. Roe, Approximate riemann solvers, parameter vectors, and difference schemes, Journal of Computational Physics 135 (2) (1997) 250 - 258. doi:10.1006/jcph.1997.5705.

URL http://www.sciencedirect.com/science/article/pii/ S0021999197957053

${ }_{545}$ [54] P. Roe, J. Pike, Efficient construction and utilization of approximate riemann solutions, Computing Methods in Applied Sciences and Engineering 6 (1984) 499-518.

[55] A. Harten, P. Lax, B. Leer, On upstream differencing and godunov-type schemes for hyperbolic conservation laws, SIAM Review 25 (1) (1983)

35-61. arXiv:http://epubs.siam.org/doi/pdf/10.1137/1025002, doi: $10.1137 / 1025002$.

URL http://epubs . siam .org/doi/abs/10.1137/1025002

[56] S. Davis, Simplified second-order godunov-type methods, SIAM Journal on 口. Scientific and Statistical Computing 9 (3) (1988) 445-473. arXiv:http: 
[57] B. Einfeldt, On godunov-type methods for gas dynamics, SIAM Journal on

[60] F. Dubois, G. Mehlman, A non-parameterized entropy correction for roe's approximate riemann solver, Numerische Mathematik (Germany) 73 (2)

[61] F. Rieper, A low-mach number fix for roes approximate riemann solver, Journal of Computational Physics 230 (13) (2011) 5263 - 5287. doi:http://dx.doi.org/10.1016/j.jcp.2011.03.025.

UR S0021999111001689

[62] P. Lax, B. Wendroff, Systems of conservation laws, Communications on п Pure and Applied Mathematics 13 (2) (1960) 217-237. doi:10.1002/cpa. 3160130205 .

580

[63] R. Richtmyer, A survey of difference methods for non-steady fluid dynamics, Tech. rep. (1962). doi:10.5065/D67P8WCQ. 
URL http://nldr.library.ucar.edu/repository/collections/ TECH-NOTE-000-000-000-050

[70] B. V. Leer, Towards the ultimate conservative difference scheme. iv. a new approach to numerical convection, Journal of Computational Physics 23 (3)

[66] E. Toro, The weighted average flux method applied to the euler equations Philosophical Transactions: Physical Sciences and Engineering 341 (1662) (1992) pp. 499-530.

URL http://www.jstor.org/stable/54156

[67] B. van Leer, Towards the ultimate conservative difference scheme i. the quest of monotonicity 18 (1973) 163-168. doi:10.1007/BFb0118673. URL http://dx.doi.org/10.1007/BFb0118673

[68] B. van Leer, Towards the ultimate conservative difference scheme.

a ii. monotonicity and conservation combined in a second-order 600 scheme, Journal of Computational Physics 14 (4) (1974) 361 - 370. doi:10.1016/0021-9991(74)90019-9. URL http://www.sciencedirect.com/science/article/pii/ 0021999174900199

[69] B. V. Leer, Towards the ultimate conservative difference scheme iii. upstream-centered finite-difference schemes for ideal compressible flow, Journal of Computational Physics 23 (3) (1977) 263 - 275. doi:10.1016/ 0021-9991(77)90094-8.
(1977) 276 - 299. doi:10.1016/0021-9991(77)90095-X. 
[71] B. V. Leer, Towards the ultimate conservative difference scheme. v. a second-order sequel to godunov's method, Journal of Computational Physics 32 (1) (1979) 101 - 136. doi:10.1016/0021-9991(79)90145-1

[72] A. Harten, S. Osher, Uniformly high-order accurate nonoscillatory schemes. i, SIAM Journal on Numerical Analysis 24 (2) (1987) 279309. arXiv:http://epubs.siam.org/doi/pdf/10.1137/0724022, doi: 10.1137/0724022.

URL http://epubs .siam.org/doi/abs/10.1137/0724022

[73] C. Machado, C. Ikoku, Experimental determination of solids fraction and minimum volumetric requirements in air and gas drilling, Journal of Petroleum Technology 34 (11) (1982) 2645-2655.

[74] R. C. Temple, R. Watson, M. Adewumi, et al., An experimental study of pneumatic transport of solids in a vertical wellbore annulus, in: SPE Eastern Regional Meeting, Society of Petroleum Engineers, 1996.

${ }_{625}^{6}$ [75] R. Temple, An experimental study of pneumatic transport of solids in a vertical wellbore annulus, Pennsylvania State University, 1995.

URL https://books . google.com/books?id=4FpQAQAAIAAJ

[76] R. Angel, Volume requirements for air or gas drilling, Petroleum Transactions, AIME 210 (1957) 325-330. 